\title{
The Effect of a Stimulation Pattern on Force and Fatigue of Paralyzed Human Quadriceps
}

\author{
Henry M. Franken', Student Member, IEEE, André van Harn'1, Peter H. Veltink' ${ }^{1}$ Member, IEEE, \\ Morten Thomsen' ${ }^{2}$ and Herman B.K. Boom', Member, IEEE
}

\begin{abstract}
'Biomedical Technological Institute, University of Twente, 7500 AE Enschede, The Netherlands ${ }^{2}$ Dept. of Medical Informatics and Image Analysis, University of Aalborg, 9220 Aalborg, Denmark
\end{abstract}

Abstract- The effects of several stimulation patterns at constant duty cycle on isometric and isokinetic knee torque development and fatigue-induced torque decline in electrically stimulated paralyzed human quadriceps were studied. The benefit of optimizing the interpulse intervals (IPIs) in comparison to a repetitive train (constant IPIs) increased with the number of stimulation pulses. Application of an optimized pattern on sustained intermittent stimulation resulted in higher torque-time integral (TTI) per cycle. The overall loss had a typical exponential decay reaching asymptotic values.

\section{INTRODUCTION}

Functional Electrical Stimulation (FES) of paralyzed muscles for the restoration of motor tasks is hampered by problems resulting from, e.g., poor reachability of muscles, and a fast onset of fatigue. These problems result in poor controllability of FES-induced movements, especially in applications using surface electrodes.

Many studies have been performed to circumvent these problems, although the majority were focused on force and fatigue in mammalian muscle. Using doublets or triplets resulted in optimal force [1] and postponed the onset of fatigue [2] in comparison to a repetitive train with constant interpulse intervals (IPIs). Yet, it is meaningful to try out these resuits on paralyzed human muscle.

In the current paper the influence of several stimulation patterns on force and fatigue of quadriceps in paraplegics was investigated. Force and fatigue in FES-applications are mainly determined by the number of pulses in a stimulation burst [3]. It was therefore investigated whether it was worthwhile to optimize the interpulse intervals in a burst with constant number of pulses and burst time to achieve maximal knee torque-time integral. Furthermore, it was investigated whether an optimized pattern remained optimal during a prolonged period of fatiguing cyclical on/off stimulation.

\section{METHODS}

\section{A. Subjects}

Four spinal cord injured subjects participated in this study. All had normal excitable quadriceps muscles and had been enrolled in a FES training program of at least 6 months.

\section{B. Experimental Set-Up}

The experimental set-up is shown in Fig. 1. During isometric measurements the knee-angle was fixed at $50^{\circ}$, at which the torque-angle relation revealed its optimum [3]. During isokinetic measurements the angular range was limited by anatomical restrictions of the lower leg, restrictions imposed by the dynamometer bench, and the desire to maintain a constant cycle time of $2 \mathrm{~s}$ during fatiguing contractions, comparable to a walking cycle.

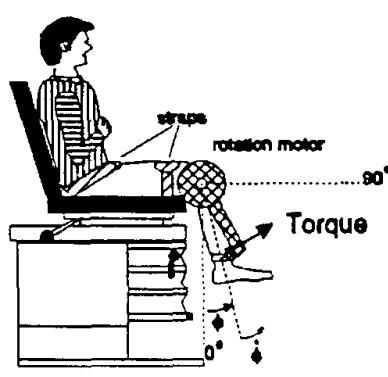

Fig. 1: Schematic of experimental sel-up. Knee angle ( $\phi)$ and velocity * $(\dot{\phi})$ are defined positive in extension motion with zero as indicated. Subject is strapped at the hip and knee to measure knee torque (at the tibia) only and to ensure static position of the body.

\section{Stimulation Patterns}

Pulsewidth (PW $=500 \mu \mathrm{s})$ and amplitude $(A=100 \mathrm{~mA})$ were set to obtain maximal recruitment, the burst time (BT) was equal to $640 \pm 20 \mathrm{~ms}$, and three sets of pulses were used $\left(n_{\mathrm{p}}=20,24\right.$, and 32$)$. For each $n_{\mathrm{p}}, 1$ repetitive train $(r), 1$ doublet of $10 \mathrm{~ms}(d), 1$ triplet of $2 \times 10 \mathrm{~ms}(t)$, and 3 exponential pattems $(e I-e 3)$ were used for investigation of torque development (TD). Exponential patterns began with 1 short IPI (10ms), while the remaining IPIs increased exponentially with a certain time constant to an asymptotic value. The repetitive train and triplet with $n_{p}=32$ were used in fatigue $(F)$ trials.

\section{Protocol}

a) In the TD trials each pattern was applied for 5 times in random order. Fatigue was minimized by taking a cycle time of $4 \mathrm{~s}$ and the course of fatigue was monitored during each TD trial.

b) Two $F$ trials were performed for both isometric and isokinetic measurements. The cycle time was $2 \mathrm{~s}$. A fatigue trial lasted 600 s with 50 minutes of recovery.

Torque, angle and angular velocity were sampled at $500 \mathrm{~Hz}$ and stored on disk for off-line analysis. 


\section{E. Off-Line Data Analysis}

The active torques measured during the TD trials were corrected for the effects of fatigue and averages of 5 responses were determined. Torque-time integral (TTI) and TTI normalized to the TTI obtained from the repetitive train (NormTTI) were derived. From active torques measured during the $F$ trials the TTI per stimulation cycle was determined.

\section{RESULTS}

\section{A. Torque Development Trials}

Typical averaged isometric torque responses of patterns with $n_{\mathrm{p}}=24$ (Fig. 2) and $n_{\mathrm{p}}=32$ (Fig. 3) are shown. The higher torque produced at the beginning of an optimized pattern could only be maintained when using $n_{p}=32$. Corresponding NormTTIs are shown in Fig. 4-5. Isokinetic measurements showed similar results.

\section{B. Fatigue Trials}

Fig. 6 shows an exponential isometric torque decline for repetitive train and triplet pattern of 32 pulses. TTI of the triplet pattern $(t)$ remained higher during the course of sustained stimulation. Similar results were found on isokinetic measurements, although TTI decreased faster for both patterns, which is caused by the influence of velocity [3].

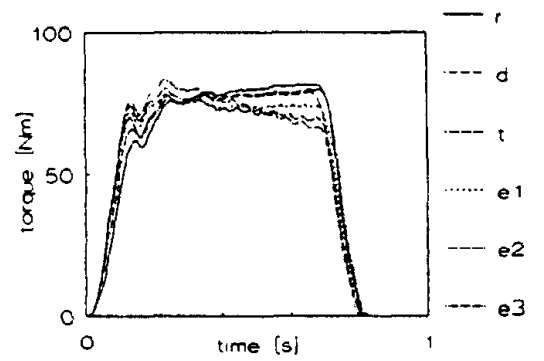

Fig. 2: Isometric torque responses of patterns with 24 pulses.

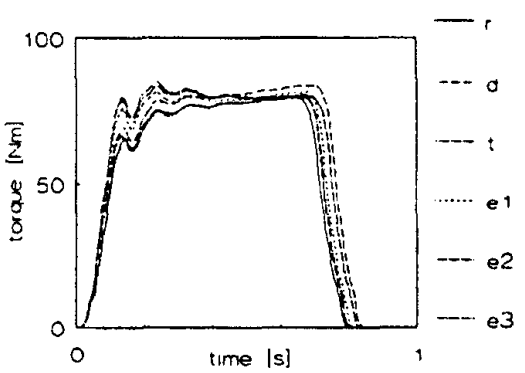

Fig. 3: Isometric torque responses of patterns with 32 pulses.

\section{IV.CONCLUSION AND Discussion}

The benefit of optimizing the IPIs in a stimulation pattern within boundary conditions is greater for a higher number of pulses, indicating that a smaller average IPI or higher average stimulation frequency is needed. During prolonged stimulation the triplet continues having a higher TTI, although the course of decline does not change. Time constants and asymptotes are similar when normalized to the maximum in a trial. Similar results are obtained from isokinetic measurements. However, the decline is faster in comparison to isometric torque, which is caused by the influence of velocity [3].

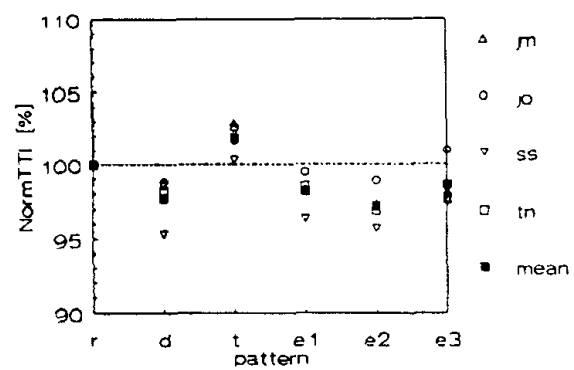

Fig. 4: Normatized TTIs: repetitive train $(r)=100 \%, n_{p}=24$.

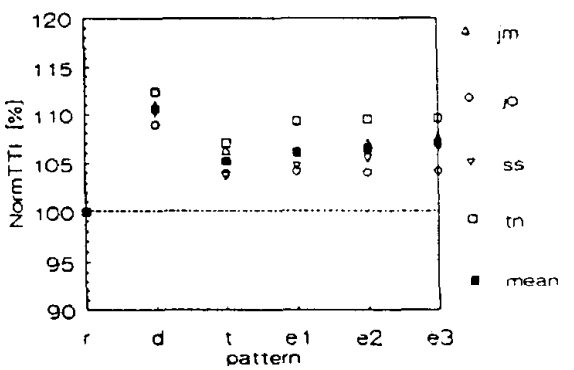

Fig. 5: Normalized TTls: repetitive train $(r)=100 \%, n_{p}=32$.

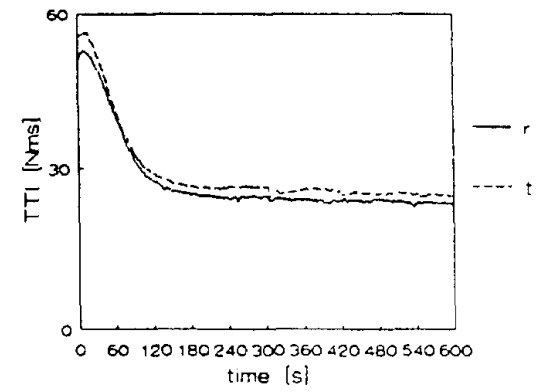

Fig. 6: Isometric TTI decline during prolonged stimulation with repetitive train (r) or triplet (l) pattern $\left(n_{p}=32\right)$.

\section{REFERENCES}

(1) F.E. Zajac and J.L. Young: Properties of stimulus trains producing maximum tension-time area per pulse from single motor units in medial gastrocnemius muscle of the cat. J. of Neuroph., Vol43, 5:1206-1220, 1980. [2] L. Bevan, Y. Laouris, R.M. Reinking and D.G. Stuart: The effect of the stimulation pattem on the fatigue of single motor units in adult cats. $J$. of Neuroph., 449:85-108, 1992.

[3] H.M. Franken, P.H. Veltink, M. Fidder and H.B.K. Boom: Fatigue of intermittently stimulated quadriceps during imposed cyclical lower leg movements. Inr. J. Elec. \& Kin., accepted, 1993. 